\title{
A Series of Endoscopic Lumbar Foraminoplasty Treatment for Radiculopathy in an Obese and Physically Disabled Patient: Case Study
}

\author{
Kai-Xuan Liu, MD, PhD
}

Endoscopic lumbar foraminoplasty is a minimally invasive spine surgery that can be performed under local anesthesia in outpatient settings. The main purpose of the technique is to relieve the compressed foraminal nerve by enlarging the neural foramen. Under direct view provided by fluoroscopy and with the assistance of an endoscope, endoscopic spine surgeons can use the technique to free the nerve by removing compression caused by disc herniations, facet joint osteophytes, scar tissue, as well as excessive ligaments.

Because of its minimally invasive nature and effectiveness in relieving symptoms associated with nerve compression, the technique has gained growing attention among endoscopic spine surgeons, as well as patients who need interventional pain management for their spinal conditions. In the past 2 decades, the technique has been modified to treat a wide range of spinal conditions, including disc herniation $(1,2)$, foraminal stenosis (3-5), and degenerative spondylolisthesis (6). The technique has also been used to treat failed back surgery (5), as well as lumbar radiculopathy occurring after instrumented lumbar spinal fusion (7). Published results suggest that in carefully selected patient population, endoscopic lumbar foraminoplasty can achieve results comparable to traditional open surgery, but with significantly faster recovery and fewer complications.

Despite the increasing number of publications on endoscopic lumbar foraminoplasty, reports on the usage of the technique in treating obese patients whose physical mobility has been impaired because of physical injuries, chronic pain, and failed surgery are limited.

Author for correspondence: Kai-Xuan Liu, MD, PhD Address: Atlantic Spine Center, West Orange, NJ 07052

E-mail: kliu@atlanticspinecenter.com
The purpose of this article is to present a case report of a male patient who was disabled because of back and leg pain caused by workplace injuries, and whose previous back surgeries not only had failed to effectively relieve the pain, but also distorted the anatomy of his spine. Limitations and potentials of endoscopic spine foraminoplasty in treating complex back pain syndromes will also be discussed.

\section{CASE REPORT}

\section{Medical History}

A 58 year old male patient endured a work related back injury in 2010. He suffered severe lower back and leg pain and was subsequently diagnosed with lumbar disc herniation and stenosis. To relieve the pain, the patient underwent 3 posterior lumbar spine surgeries from 2011 to 2013 at a surgical center in New York. The techniques used by the spine surgeon included microscopic laminectomy, discectomy, facetectomy, foraminotomy, as well as spinal fusion using autologous local bone graft and allograft material at the L3-L4, L4-L5, and L5-S1 levels.

The surgeries unfortunately failed to effectively relieve the pain. After the initial and revision surgeries, the patient experienced bilateral leg and foot numbness, burning pain, and weakness in both knees and ankles. Eventually, the patient lost his ability to walk or even stand up because of residual and recurrent pain and muscle weakness.

In 2014 the patient went to the spine center where the author works to seek pain treatment. Magnetic resonance imaging (MRI) showed bilateral foraminal stenosis and bone grafts at multiple disc levels, including L3-L4, L4-L5, and L5-S1. The stenosis was combined with disc space narrowing and disc herniation at the L3-L4 level; vertebral osteophytes and disc space narrowing at L4-L5; osteophytes, disc herniation, and disc space narrowing at L5-S1 

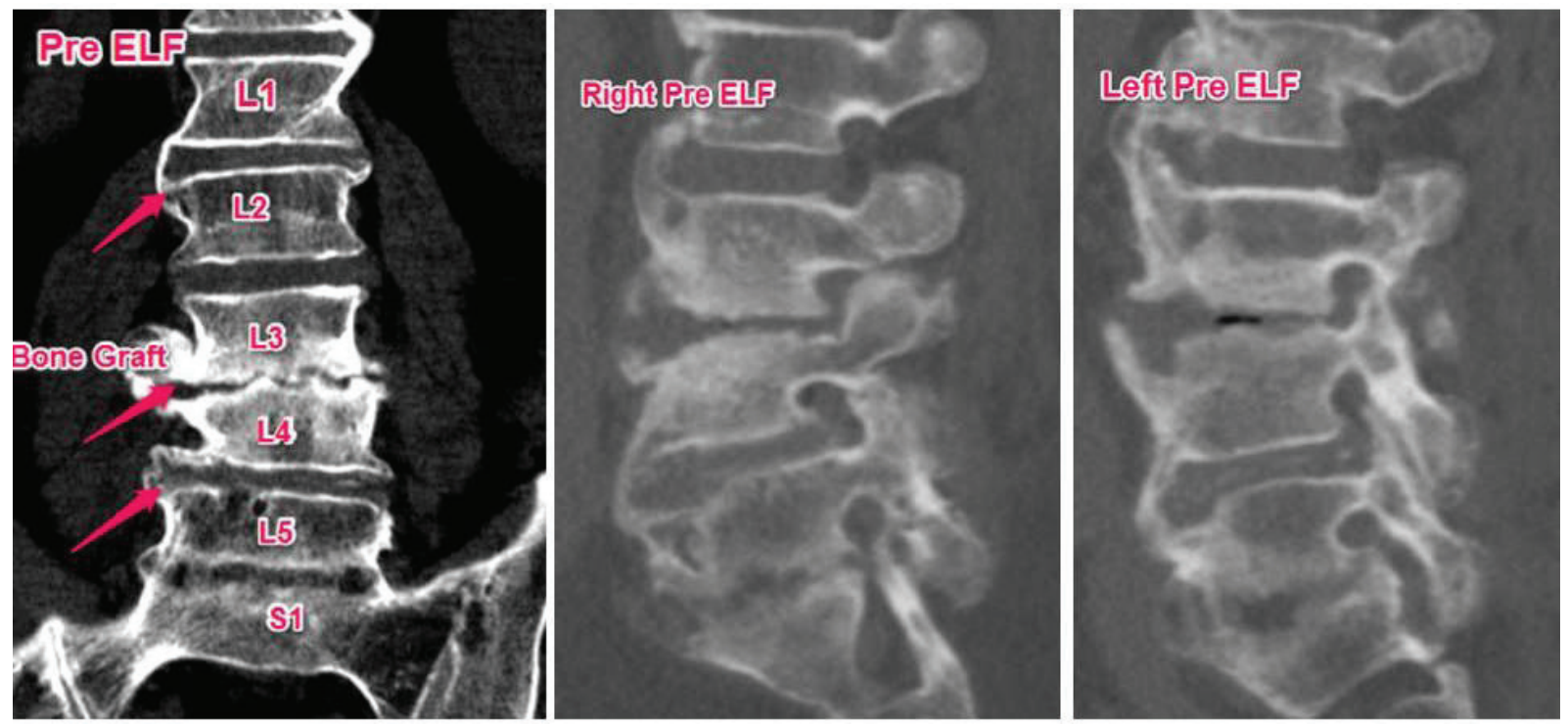

Fig. 1. MRI findings of the spine condition at L1-S1.

level. Vertebral osteophytes were also shown at L2 vertebra (Fig. 1).

Traditional pain treatments, including pain medications, failed to effectively relieve the pain -- the patient described a pain score of 10 despite the pain medications. And the patient refused to undergo another traditional spine surgery.

After consulting with the author about available treatment options, the patient chose to undergo endoscopic lumbar foraminoplasty and discectomy.

\section{STUDY DESIGN}

The patient was extremely obese (with a body mass index of 48.1), and general anesthesia was deemed too risky. Local anesthesia combined with intravenous sedation was considered a safe option for the patient. However, under local anesthesia the patient was not able to stay still on the operation table for longer than one hour, yet the complexity of his spinal condition required an extensive surgery that could last much longer than one hour.

With the patient's consent, the author designed a stage-by-stage serial surgical treatment for the patient (Table 1). From February 2014 to April 2015, a total of 8 endoscopic lumbar foraminoplasty and discectomy procedures were performed.

\section{Surgical Technique}

The surgery was performed under aware-state with the patient lying in the prone position on a flexed radiolucent table. Under local anesthesia (a combination of .5\% lidocaine with epinephrine) and intravenous sedation, the patient was awake and was able to communicate with the surgeon throughout the surgery.

Under fluoroscopy guidance, an 18-guage needle was inserted towards the target spinal foramen via a posterolateral approach. The needle was used to inject dye into the intervertebral disc to evaluate the disc's integrity, to perform discography, and to develop an optimal inserting route for an endoscope. For L3L4 and L4-L5 levels, the needle was inserted towards the middle pedicle line. For L5-S1, the fluoroscopy was adjusted to ensure that the anterior-posterior (AP) view was parallel to the L5-S1 endplate, and the needle was inserted towards the junction between superior articular process (SAP) and the iliac crest.

After landing at the ideal position, the needle was replaced with a long guide wire. Over the guide wire, a sequential dilator was used to establish an inserting path for an endoscopic cannula and a safe working space. After an ideal path has been established, a tubular retractor was placed and the dilator was re- 
moved. An endoscope with a working channel was then inserted through the tubular retractor, and the remaining procedure was carried out under direct visualization.

In general, a bone shaver was used to shave the superior articular facet and to undercut the ventral part of the ascending facet to enlarge the foramen. The disc herniations were removed using a pituitary rongeurs and were pulled out with a grasper. The scar tissue was largely removed using a bipolar cutter and forceps, and the osteophytes were removed with a diamond burr.

\section{RESULTS}

At the end of the serial treatment, the patient was able to stand up and walk with a walker, feeling minimum pain and numbness in the legs and feet. The patient's muscle strength in knees and ankles had returned to normal at the last follow-up, and his leg pain, numbness, and muscle weakness improved $80 \%$. There were no complications during or after the surgery.

Post surgery MRI showed that the patient's foramina at L3-L4, L4-L5, and L5-S1 were opened up (Fig. 2).

\section{DISCUSSION}

The patient's spine anatomy was completely distorted from the previous 3 open spine surgeries and fusion. Disc herniations, bone spurs, and bone grafts caused nerve compression at multiple locations (L3-L4, L4-L5, and L5-S1). The patient was overweight partly because of inadequate exercise caused by the severe leg and foot pain and numbness. After the initial 3 open spine surgeries, the patient did not want to undergo another round of open surgery. Other conservative treatment options, including pain medications and physical therapy, failed to effectively relieve the pain. The patient's pain score (visual analog scale) remained 10 even when he was taking pain medications. The patient was open to endoscopic lumbar foraminoplasty largely because of its minimally invasive nature.

Given the complexity of the patient's spinal condition and the fact that the patient wasn't able to stay still for longer than one hour at a time on the operation table, it's impossible to address all the
Table 1. A step-by-step surgical plan.

\begin{tabular}{|c|c|c|c|c|}
\hline Date & Side & ELF & Revision & Reason \\
\hline $02 / 19 / 2014$ & Left & L3-L4, L4-L5 & & $\begin{array}{c}\text { Pain in the } \\
\text { thigh and shin; } \\
\text { knee weakness }\end{array}$ \\
\hline $03 / 17 / 2014$ & Left & L5-S1 & & $\begin{array}{c}\text { Foot numbness } \\
\text { and weakness }\end{array}$ \\
\hline $05 / 01 / 2014$ & Right & L3-L4, L4-L5 & & $\begin{array}{c}\text { Pain in the } \\
\text { thigh and shin; } \\
\text { knee weakness }\end{array}$ \\
\hline $06 / 06 / 2014$ & Right & L5-S1 & & $\begin{array}{c}\text { Foot numbness } \\
\text { and weakness }\end{array}$ \\
\hline $01 / 08 / 2015$ & Right & & L4-L5, L5-S1 & $\begin{array}{c}\text { Inadequate pain } \\
\text { relief }\end{array}$ \\
\hline $02 / 19 / 2015$ & Right & & L3-L4 & $\begin{array}{c}\text { Inadequate pain } \\
\text { relief }\end{array}$ \\
\hline $04 / 02 / 2015$ & Left & & L5-S1 & $\begin{array}{c}\text { Inadequate pain } \\
\text { relief }\end{array}$ \\
\hline $04 / 02 / 2015$ & Left & & L3-L4 & $\begin{array}{c}\text { Return of pain } \\
\text { in the thigh and } \\
\text { shin }\end{array}$ \\
\hline
\end{tabular}

spinal conditions through a single surgery. A serial, multistage surgical treatment offered an option to decompress the nerves 1 or 2 at a time. As expected, the patient's condition gradually improved. After the final surgery, the patient was able to get out of his wheelchair and walk around with a walker.

Recurring pain and inadequate pain relief following spine surgery is a common concern among surgeons as well as patients. The cause of the recurring pain is multi-faceted, including but not limited to inadequate decompression in the original surgery, pseudoarthosis following bone grafting and spinal fusion, and post surgery disc reherniation. Reherniation itself is a complex issue. It could be a result of many different factors, including, again, inadequate decompression in the original surgery, the degenerative nature of the disc especially in elderly patients, and the patient's ever changing health conditions.

It's challenging, if not impossible, to completely prevent disc reherniation. Compared with traditional open surgery, minimally invasive techniques, such as endoscopic lumbar foraminoplasty, offer a less traumatic revision option to the patient. This case report shows that endoscopic lumbar foraminoplasty can be repeatedly performed on the same patient safely without complications. Its effectiveness in relieving pain combined with its minimally invasive nature makes the technique much 

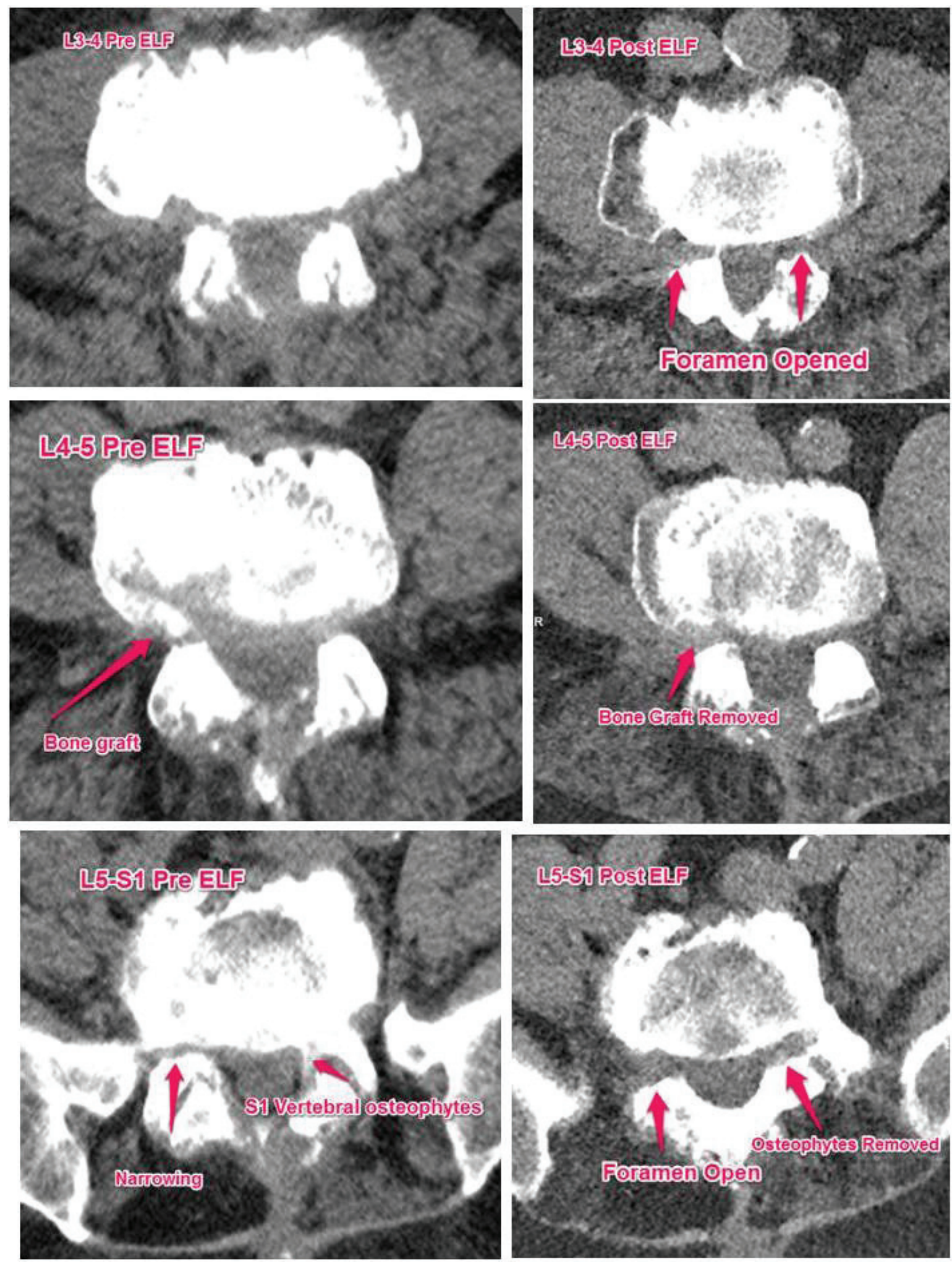

Fig. 2. Comparison between pre- and post-surgery MRI findings. 
more acceptable, and necessary revision procedures less daunting, to the patient than open spine surgery.

As reported by Telfeian and colleagues, the more levels of fusion involved and the more levels of foraminoplasty required, the more challenging it is to improve the patient's condition. In our case, the patient needed 4 foraminoplasty revisions to further relieve the pain caused by inadequate decompression from the initial surgery and from recurring herniation. Part of the reason for the inadequate decompression was the patient's inability of staying still on the surgical table for longer than one hour. However, the minimally invasive nature made the revisions acceptable to the patient. At the end of the treatment, the patient was satisfied with the result and was open to more similar procedures if needed again in the future.

\section{Potential Applications}

Endoscopic lumbar foraminoplasty can be used to effectively treat lumbar radiculopathy caused by disc degeneration-induced disc space narrowing, facet hypertrophy, vertebral osteophyte, disc herniation, spondylolisthesis, as well as failed back surgery syndrome.

\section{Limitations}

Endoscopic lumbar foraminoplasty is associated with a deep learning curve. The use of the surgical tools under endoscopic control may be challenging to spine surgeons without adequate training.

\section{CONCLUSION}

Endoscopic lumbar foraminoplasty can be used to safely and effectively treat lumbar radiculopathy that has failed to respond to conservative treatments. It offers a less traumatic treatment option to patients than traditional open spine surgery, and it can be safely performed on the same patient repeatedly.

\section{Acknowledgments}

The author did not receive outside funding to support the study and preparation of this report. The author declares no commercial association that may pose a conflict of interest in connection with this report. The author thanks Dr. Yanni Wang for providing editorial assistance in preparing this manuscript.

\section{REFERENCES}

1. Choi G, Lee SH, Lokhande P, Kong BJ, Shim CS, Jung B, Kim JS. Percutaneous endoscopic approach for highly migrated intracanal disc herniations by foraminoplastic technique using rigid working channel endoscope. Spine (Phila Pa 1976) 2008; 33:E508-E515

2. Choi KC, Park CK. Percutaneous endoscopic lumbar discectomy for L5-S1 disc herniation: Consideration of the relation between the iliac crest and L5-S1 disc. Pain Physician 2016; 19:E301-E308.

3. Ahn Y, Oh HK, Kim H, Lee SH, Lee HN. Percutaneous endoscopic lumbar foraminotomy: An advanced surgical technique and clinical outcomes. Neurosurgery 2014; 75:124-133.

4. Li ZZ, Hou SX, Shang WL, Cao Z, Zhao HL. Percutaneous lumbar foraminoplasty and percutaneous endoscopic lumbar de- compression for lateral recess stenosis through transforaminal approach: Technique notes and 2 years follow-up. Clin Neurol Neurosurg 2016; 143:90-94.

5. Knight MT, Jago I, Norris C, Midwinter L, Boynes C. Transforaminal endoscopic lumbar decompression \& foraminoplasty: A 10 year prospective survivability outcome study of the treatment of foraminal stenosis and failed back surgery. Int J Spine Surg 2014; $1: 8$.

6. Jasper GP, Francisco GM, Telfeian AE. Transforaminal endoscopic discectomy with foraminoplasty for the treatment of spondylolisthesis. Pain Physician 2014; 17:E703-E708.

7. Telfeian AE, Jasper GP, Francisco GM. Transforaminal endoscopic treatment of lumbar radiculopathy after instrumented lumbar spine fusion. Pain Physician 2015; 18:179-184. 
